Rev. Hist., N²7, vol. 2, Julio-Diciembre 2020: 303-319

ISSN 0717-8832

https://doi.org/10.29393/RH27-19GMUP20019

\title{
Goro Miyazaki: Intriga y sospecha sobre el espionaje japonés en Chile durante la Segunda Guerra Mundial*
}

\author{
Goro Miyazaki: Intrigue and suspicion of Japanese espionage in Chile during World War II
}

Ulises Parraguez: ORCID: https://orcid.org/0000-0001-8493-7519**

Pedro lacobelli: ORCID: https://orcid.org/0000-0002-5501-8529***

\section{RESUMEN:}

Este artículo es fruto de una investigación en torno al ciudadano japonés Goro Miyazaki, residente en Chile durante la Segunda Guerra Mundial y su posterior expulsión luego de ser acusado de llevar a cabo actividades contra los intereses nacionales. Considerando esto, se presenta el estudio realizado en torno a este ciudadano japonés, en un ambiente marcado por tendencias xenofóbicas, raciales y de sospecha frente a cualquier aspecto que tensionara la declarada neutralidad chilena en el conflicto mundial. Si bien no es posible declarar de manera terminante la calidad de espía de Miyazaki, sí se puede destacar la particularidad de su caso, en la medida que fue el único japonés expulsado en este momento. El trabajo se sustenta en la revisión y análisis de fuentes escritas, archivos notariales y gubernamentales, destacando la defensa del propio Miyazaki para evitar su expulsión.

Palabras clave: Goro Miyazaki, espionaje, Segunda Guerra Mundial, Chile.

\section{ABSTRACT:}

This article is the result of a research on the Japanese citizen Goro Miyazaki, a resident of Chile during World War II and his subsequent expulsion after being accused of carrying out activities against national interests. Considering this, the study carried out around this Japanese citizen, in an environment marked by xenophobic tendencies, racial and suspicion against any aspect that strained the declared Chilean neutrality in the world conflict, is presented. While it is not possible to strictly state Miyazaki's spy quality, if one can highlight the particularity of his case, to the extent that he was the only Japanese expelled at this time. The work is based on the review and analysis of written sources, notary and government files, highlighting Miyazaki's defense to prevent his expulsion.

Keywords: Goro Miyazaki, espionage, World War II, Chile

Recibido: octubre 2019

Aceptado: julio 2020

\footnotetext{
* El presente artículo es producto del trabajo realizado en el marco del proyecto Fondecyt Iniciación $\mathrm{N}^{\circ} 11160011$ "Chile y Japón, de la neutralidad al quiebre de relaciones, 1941-1942".

** Licenciado en Historia, Pontificia Universidad Católica de Chile. Profesor de Historia, Geografía y Ciencias Sociales, Universidad Alberto Hurtado.

*** Doctor en Historia, The Australian National University. Profesor asistente Pontificia Universidad Católica de Chile y director Centro UC Estudios Asiáticos, Chile. Email: piacobel@uc.cl
} 


\section{Introducción}

Al tiempo del imparable avance japonés en su guerra contra China (1937-1945) y la consolidación del engrandecimiento del Reich alemán en Europa del este, en Santiago de Chile se levantaba una nueva empresa japonesa. El 31 de enero de 1940, se estableció la "Sociedad Comercial Chileno-Oriental", cuyos firmantes fueron Yonosuke Hagiwara, Sueyuki Nakatsuka y Goro Miyazaki. Esta nueva sociedad tenía como giro, al igual que otras de reciente creación, la importación y exportación de artículos varios, además de "cualquier bien que los socios estipulasen"1. En sus estatutos se estipuló que la Sociedad tendría una vigencia de cinco años, con la posibilidad de extenderla si así lo deseaban los socios. Esta sociedad adquirió mayor relevancia al constituirse como el brazo comercial del gigante japonés Mitsui Bussan Kaisha en el país. Goro Miyazaki -el capitalista principal de la nueva sociedad-trabajó en Buenos Aires representando a la firma japonesa Nambei Importaciones S.A. hasta 1940, año en el que migra a Chile. En términos comerciales la llegada de Goro Miyazaki se enmarca en un periodo de gran afinidad económica y social entre Chile y el Imperio de Japón. Sin embargo, el ataque japonés a Pearl Harbor, en diciembre de 1941, puso en alerta no solo a las autoridades estadounidenses, sino también a todo el continente americano de la presencia nipona en el continente. Chile, al igual que el resto de las repúblicas americanas, fue parte de una ola de sospechas e intrigas que rodearon a las comunidades de migrantes de los países del Eje. En este nuevo contexto, Goro Miyazaki y las actividades de la Sociedad Comercial Chileno-Oriental fueron objeto de escrutinio -al igual que los ciudadanos alemanes e italianos- cuya intensidad fue aumentando a medida que avanzaba la guerra, la neutralidad chilena se debilitaba y se descubrieron pequeñas redes de transferencia de información operadas por ciudadanos alemanes y chileno-alemanes ${ }^{2}$. Finalmente, Goro Miyazaki, a pesar de la rápida y contundente defensa del ministro japonés en el país, Kiyoshi Yamagata, fue expulsado bajo sospecha de espionaje en agosto de 1942.

Este artículo vincula el caso de Goro Miyazaki con la política y sociedad americana en tiempos de la Segunda Guerra Mundial (SGM) en distintos planos. Da cuenta de una creciente presencia comercial japonesa en el país, así como la extrapolación de temores y prejuicios raciales de parte de la población chilena hacia lo japonés. Estos temas han sido tangencialmente tratados por los estudios sobre Chile y la SGM, y Chile y Japón en general. En la primera de estas

\footnotetext{
1 Se formó con un capital de $\$ 120.000$ pesos, apartado en 80 mil por el señor Miyazaki y 20 mil por cada uno de los restantes. Registro de Comercio del Conservador de Bienes Raíces de Santiago, foja 196, № 151, 31 de enero de 1940.

2 Por ejemplo, se dio cuenta por la prensa de las intervenciones a las líneas de comunicaciones de la Armada, de lo cual dieron cuenta los periódicos nacionales el 15 de mayo de 1942 "Remite recortes de prensa, que se refieren a interferencia en las líneas telefónicas de la Armada", Cuaderno 16, Dirección General de Investigaciones e Identificación, Departamento 50, Archivo Nacional de Chile, 15 de mayo de 1942. Disponible en: http://www.archivonacional.cl/sitio/Contenido/Institucional/82968:Cuadernos-del-11-al-23 Sobre espionaje alemán en Chile ver Farías, Víctor. 2000. Los Nazis En Chile, Hong Kong, Editorial Wide Chance.
} 
literaturas, la relación con Japón es vista sucintamente por autores que colocan el foco gravitante del periodo en las relaciones entre Chile y EE. UU. ${ }^{3}$ En la segunda, sobresalen estudios generales sobre las relaciones entre Japón y Chile como los de Álvaro Castellón y José Miguel Pozo, y Cesar Ross, quienes a partir de un trabajo en los archivos del Ministerio de Relaciones Exteriores de Chile dan cuenta principalmente de la relación diplomática y económica ${ }^{4}$. El cruce entre espionaje y sociedad (o seguridad nacional y lo japonés en Chile) es tratado con profundidad solo en la tesis doctoral de Mauricio Paredes, quien estudia la relegación nipona en Chile luego de la ruptura de relaciones en 1943 y sus antecedentes inmediatos, en los cuales nombra a Miyazaki y las acusaciones que pesaron sobre él ${ }^{5}$. Empero, el problema del espionaje japonés en Chile y su significación en el contexto social no ha sido aún desarrollado ${ }^{6}$. Este texto, viene a introducir este problema a través del caso específico de Goro Miyazaki.

La Segunda Guerra Mundial constituyó en Chile y el mundo una experiencia única, que llevó al borde las lealtades y nociones identitarias en diversas comunidades nacionales. Los ciudadanos del Eje, en particular la pequeña comunidad japonesa en Chile, fue emplazada a un espacio marginal marcado por la sospecha de espionaje. El caso de Goro Miyazaki se inserta dentro de esta vorágine coyuntural de miedo a nivel mundial y continental, que creció intempestivamente frente a la alianza germano-nipona de 1937 y, sobre todo, a lo impredecible y sorpresivo que resultó el ataque japonés a EE. UU. y las acciones tomadas posteriormente para frenar la penetración de agentes externos en las naciones americanas. Para efectos de este escrito, se considerará el contexto de la participación de las comunidades japonesas en el país con énfasis en el ámbito comercial y la figura de Goro Miyazaki en ella. Luego, se emplazará

\footnotetext{
${ }^{3}$ Entre los principales autores ver Fermandois, Joaquín. 1988. "Guerra y Hegemonía 1939-1943. Un Aspecto de Las Relaciones Chileno-Norteamericanas," Historia 23, no. 1, pp. 5-51; Nocera, Rafaelle. 2006. Chile y La Guerra 19331943, Santiago, LOM editores.; Barros Van Buren, Mario. 1998. La Diplomacia Chilena En La Segunda Guerra Mundial, Santiago, Empresa Editoria Arquen.

${ }^{4}$ Castellón, Alvaro. y Pozo, José Miguel. 1997. "Historia de las Relaciones Bilaterales Chile y Japón," en Oscar Pinochet de la Barra (ed). Chile y Japón Un Siglo de Amistad, ed., Santiago, Comisión Chilena de Celebración del Centenario de las Relaciones Chile-Japón, pp. 129-60; Ross, César. 2013. "Auge y Caída de Japón En Chile, 18971943," Estudios Políticos 43, pp. 156-179; lacobelli, Pedro. 2016. "La 'neutralidad' chilena en la segunda guerra mundial (1939-1943): Un Análisis Historiográfico con énfasis en la literatura sobre las relaciones Chile-Japón,"en Revista de Historia y Geografía № 34, pp. 95-108.

5 Paredes, Mauricio. 2012. Nacionalismo, Seguridad y Presión Internacional: La Relegación de Japoneses En Chile Durante La Segunda Guerra Mundial, Santiago, Universidad de Chile. El vínculo entre sociedad y presencia japonesa ha sido estudiado por lacobelli, Pedro. y Camino, Nicolás. 2019. "Diplomacia pública japonesa en la prensa chilena Durante la segunda guerra mundial," Cuadernos de Historia, 2019, Santiago, pp. 73-97 ; lacobelli, Pedro. 2019. "Reconsiderando la neutralidad chileno-argentina: Prensa y diplomacia japonesa durante La Conferencia de Río de Janeiro, 1942," Ayer., vol. 119, no. 3, Madrid, pp. 137-163.

${ }^{6}$ Existen estudios de espionaje japonés en el continente pero que no informan sobre el caso chileno, ver, por ejemplo, Rodao, Florentino. 2012. Franco y El Imperio Japonés, Barcelona, P@, Osprey Publishing; Matthews, T. 2010. Spies for Nippon: Japanese Espionage Against the West, 1939-1945, Londres, Robert Hale \& Company.
} 
las acusaciones de espionaje de Miyazaki y posterior expulsión en el contexto de inminente ruptura de relaciones y reciente descubrimiento de redes de espionaje alemán. Finalmente se entregarán las conclusiones de este trabajo.

\section{Presencia japonesa en Chile al tiempo de Pearl Harbor}

La presencia japonesa en Chile ha sido descrita como menor (en comparación con la de otros países americanos como Brasil y Perú), dispersa en el territorio, y que tendió a mezclarse con la población chilena siendo comunes los matrimonios interétnicos que terminaron por socavar los rasgos identitarios propios como son el idioma, las tradiciones y religión ${ }^{7}$. Las cifras globales de inmigración dan cuenta de aquello, pues mientras en Brasil para el periodo 1900 a 1940 habían ingresado cerca de doscientos mil japoneses y en Perú poco más de treinta mil, en Chile -según estadística nipona- habían entrado quinientos migrantes ${ }^{8}$. Pero esta imagen más bien monolítica, de insuficiencias e irrelevancia de la comunidad nikkei puede no dejar ver otra realidad que se evidenció en la década del treinta y que permite establecer el contexto de la llegada de Goro Miyazaki a Chile.

En primer lugar, las relaciones entre Chile y Japón gozaban de un muy buen momento político y público. De hecho, como recientemente se ha expuesto, hacia los meses anteriores al ataque sobre la escuadra estadounidense en Pearl Harbor, en Chile se difundió una imagen positiva de Japón, en parte producto de una agenda de diplomacia pública japonesa activa, en parte al interés real en grupos de la población por el modelo de desarrollo japonés (un primer caso de "milagro económico") ${ }^{9}$. Se puede decir que la prensa con una línea editorial cercana a las derechas chilenas (pensamos en, por ejemplo, El Mercurio, El Diario Ilustrado, El Chileno, entre otros) observaban a Japón a través de un lente que matizaba su cultura, modernidad y "pacifismo". La prensa oficialista mantuvo también una visión positiva de Japón pero con mayores reparos en la medida que la guerra sobre China no culminaba y las hostilidades contra EE. UU. se acentuaban ${ }^{10}$. Los vínculos positivos se estrecharon gracias a una política comercial que -a pesar del proteccionismo de la época-consiguió aumentar el intercambio de productos

\footnotetext{
${ }^{7}$ Fenómeno particularmente evidente en la segundas y terceras generaciones de chileno-japoneses. Este fenómeno ha sido estudiado por la misma comunidad nipona en Chile, como lo demuestran las obras Ferrando, María Teresa. 2004. Al Otro Lado Del Pacífico: Japoneses En Chile, 1900 - 1960, Santiago, Ograma,.; Takeda M., Ariel. 2002. "Japanese Immigrants and Nikkei Chileans," in Encyclopedia of Japanese Descendants in the Americas, 202AD, p. 182. Como por investigadores ajenos a ella, Ross, César. 1999. "Chile y Japón: Balance de Un Siglo de Relaciones Económicas, 1897-1997," Revista Diplomacia 78; lacobelli, Pedro. 2017. Postwar Emigration to South America from Japan and the Ryukyu Islands, London, Bloomsbury Academic, capítulo 2.

${ }^{8}$ Gaimushō Ryōji lijūbu, "Waga kokumin no kaigai hatten: ijū hyakunen no ayumi (honhen) ," ed. Ministry of Foreign Affairs. 1971. Tokyo, pp. 142-43.

9 lacobelli, Pedro y Camino, Álvaro. 2019. “Diplomacia Pública Japonesa En La Prensa Chilena Durante La Segunda Guerra Mundial."

${ }^{10} \mathrm{lbid}$.
} 
entre países. Como Castellón y Pozo han estudiado, una serie de viajes e intercambios culturales permitieron estrechar estas relaciones ${ }^{11}$.

El buen momento, y el ímpetu de la diplomacia pública nipona se ven reflejada en la fundación del Instituto Cultural Chileno-Japonés el 9 de enero de 1940, el cual constituyó un peldaño relevante en el esfuerzo nipón de fortalecer los vínculos entre Japón y miembros de la alta sociedad santiaguina ${ }^{12}$. El Instituto estableció en su carta fundante tener por objetivo el "fomentar la tradicional relevancia de amistad entre Chile y Japón mediante la intensificación de su mutua cooperación cultural, científica, económica y de todo orden"13. Esto, por cierto, iba en la línea estimada desde Tokio, en particular a través de la Kokushi Bunka Shinkōkai (Asociación para la Promoción de la Cultura Internacional) en 1934, órgano coordinador de soft power japonés en el mundo ${ }^{14}$. Para el caso chileno, el Instituto procuró desarrollar actividades que permitieran mayor conocimiento de los diversos aspectos de la vida en Japón y cooperación entre países ${ }^{15}$. Un ejemplo de la compenetración entre Japón y Chile se ve en el directorio del Instituto que incorporó al rector de la Universidad Católica monseñor Carlos Casanueva, a los senadores Maximiano Errazuriz y Hernán Figueroa Anguita, y a otros importantes políticos y hombres de ciencia de la época como Desiderio García A., Guillermo Valenzuela, Walter Müller Hess, general Arturo Espinoza M., Hugo K. Sievers W, entre otros.

En segundo lugar, las buenas relaciones entre Chile y Japón se ven en la arena económica la cual, como se ha dicho, mostraba buenos números ${ }^{16}$. Según el Anuario Estadístico de Chile, para los años 1936 a 1940, las importaciones japonesas aumentaron de forma significativa. Si en 1932 las importaciones japonesas contabilizaban $\$ 1.204 .005$ pesos (de seis peniques), para el año 1937 sumaban $\$ 11.709 .044$ pesos; en 1939 \$15.310.490 pesos y 1940 \$27.911.171 pesos. El aumento del valor del comercio con Japón iba de la mano del aumento en las cantidades transadas y un mayor peso de Japón en el comercio chileno: las cifras para 1940 representaba cerca del nueve por ciento de las importaciones nacionales ${ }^{17}$.

\footnotetext{
${ }^{11}$ Castellón, Alvaro y Pozo, José Miguel. 1997. "Historia de Las Relaciones Bilaterales Chile y Japón." Sobre la política proteccionista ver Braun-Llona, J. et al.,. 1998. "Economía Chilena 1810-1995. Estadísticas Históricas", Instituto de Economía, Pontificia Universiad Católica de Chile, Santiago, 1998.

12 Veáse Estatutos Del Instituto de Cultura Chileno Japonés. 1940. Santiago, Imprenta y Litio Universo S.A.

13 lbid., p.2.

${ }^{14}$ Abel, Jessamyn. 2013. "Cultural Internationalism and Japan's Wartime Empire: The Turns of the Kokusai Bunka Shinkōkai," in Tumultus Decade: Empire, Society, and Diplomacy in 1930's Japan, p. 19.

${ }^{15}$ Estatutos Del Instituto de Cultura Chileno Japonés. 1940. pp. 3-4.

${ }^{16}$ El trabajo más completo a la fecha sobre los vínculos económicos es el elaborado por Dominique Hachette. 1997. “Relaciones Económicas Entre Chile y Japón," en Chile y Japón Un Siglo de Amistad, ed. Oscar Pinochet de la Barra, Santiago, Comisión Chilena de Celebración del Centenario de las Relaciones Chile-Japón, pp. 163-99.

17 Instituto Nacional De Estadísticas. Anuario Estadístico del Comercio de Chile. Años 1930-1941. Para un análisis de estas relaciones véase lacobelli, P. 2020. "Conservadores, Radicales y el "interés nacional": las redes de apoyo al Imperio de Japón en Chile durante la Segunda Guerra Mundial" en Izquierdas, No. 49, pp. 3242-60.
} 
Este contexto explica la llegada de Miyazaki y varios otros hombres de negocios a Chile. Sesenta y ocho japoneses arriban al país entre los años 1934 y 1941, con una concentración especial entre los años 1940-1941. De ellos, dieciséis están relacionados con empresas japonesas, sociedades o casas comerciales que iniciaron su actividad en Chile. De estas dieciséis personas, ocho se encuentran directamente vinculados con el japonés Goro Miyazaki, quien es expulsado el año $1942^{18}$. Entre esos años, se crean o establecen en el país, junto con Mitsui Bussan Kaisha y Toyo Menka Kaisha (vinculadas a Miyazaki), otras empresas tales como Casa Itoh, Casa Sanko, Sociedad Comercial Chileno Oriental, Hattori Shoten, entre otras. El momento de buenas relaciones comerciales desbordó al aspecto político y social. Esto es graficado por Akira Abe, gerente en Santiago de la flota Nippon Yusen Kaisha Line (NYK) quien, al momento de anunciar la ampliación de la flota, indicó:

"[...] la base del mutuo entendimiento entre los pueblos está en los buenos servicios de transporte de pasajeros como de productos. El viajero japonés que llega a Chile, como el chileno que va a Japón contribuyen a establecer contactos espirituales que no se olvidan fácilmente. Pero esos contactos necesitan perfeccionarse con el intercambio comercial: la compraventa de productos naturales o elaborados contribuyen en forma muy principal a desvanecer prejuicios, y a servir como medio de mejoramiento económico de cada nación"19.

En definitiva, podemos indicar que la llegada de Goro Miyazaki y otros comerciantes japoneses al país, es parte de un contexto general de migración japonesa y la expansión de Japón en Latinoamérica, vinculado a grupos comerciales, buscando fortalecer la presencia económica en América Latina. Las fuentes consultadas permiten identificar una red de personas que se vinculan con Miyazaki, relacionadas con empresas, favoreciendo un intercambio de información. En este sentido, la llegada de Miyazaki epitomiza el momento histórico de las relaciones entra ambos países, pero a la vez, da cuenta de una correlación con poseer una cierta posición privilegiada. Como se estudia en la siguiente sección, existió una relación entre Goro y la legación japonesa en Chile, dando cuenta de una red de recopilación de información autónoma y consolidada. Además, llama la atención que, tras un halo de normalidad, Miyazaki es la única persona, del universo de comerciantes japoneses, que es acusada directamente de espionaje, siendo expulsado por ello ${ }^{20}$. Su caso, como este contexto arroja, nos da cuenta de una situación extraordinaria.

\footnotetext{
18 De esas ocho personas relacionadas con Goro Miyazaki, todos fueron relegados en el transcurso del año 1943 a distintos lugares de la zona centro chilena, en localidades como Buin, Melipilla, San Francisco de Mostazal y Casablanca. Ver Ferrando, María. Teresa. 2004. Al Otro Lado Del Pacífico: Japoneses En Chile, 1900 - 1960.

${ }^{19}$ Estatutos Del Instituto de Cultura Chileno Japonés. 1940.

${ }^{20}$ Como veremos, se le acusa a partir de haber sido sorprendido con fotografías de la zona norte del país, lo que él justificó en la premisa de que se relaciona con labores propias del rubro comercial que maneja.
} 


\section{Espionaje, comerciante, amenaza: El caso de Goro Miyazaki}

La literatura sobre el espionaje japonés en Latinoamérica da cuenta de que esta se encontraba en un estado menor de consolidación, siendo subsidiada por la estructura de espionaje de Alemania. Louis Allen, por ejemplo, identifica la presencia de una red de espionaje japonés en América Latina (lo que es un elemento común entre todas las potencias beligerantes en el conflicto), pero argumenta que esta tuvo un éxito muy menor al logrado en Asia, especialmente debido a la diferencia de recursos con el Alto Comando Aliado ${ }^{21}$. Sin embargo, la presencia ubicua de japoneses en el oeste de EE. UU. y en varias localidades sudamericanas, dio pábulo a teorías conspirativas y a la difusión de recelo hacia los ciudadanos del Imperio de Japón. Durante la década de 1930, en el plano académico, revistas como Pacific Affair, Foreign Affairs y Far Eastern Survey publicaron artículos en tono de denuncia sobre la "inquietante" expansión de lo japonés en América Latina, la cual era principalmente económica, pero con implicancias políticas. Estas publicaciones alertan de que, a través de intercambios culturales, presencia de una activa comunidad de inmigrantes y la "penetración" económica nipona se produce un aumento de poder político y a través de esto una posible influencia en América Latina ${ }^{22}$. Por su parte, los medios de comunicación de masas identificaron a pescadores, migrantes, hombres de negocios y turistas nipones como parte de la vasta red de espionaje japonés ${ }^{23}$. Es útil esta categorización, en la medida que permite situar a Goro Miyazaki dentro de un contexto global, que da cuenta de los resquemores que el gobierno chileno tuvo hacia su persona.

El pensamiento antijaponés no fue un hecho aislado en tiempo de guerra; pues ya desde principios del siglo $\mathrm{XX}$, oponentes a la llegada de migrantes nipones se habían manifestado a través de varios medios. Por ejemplo, diarios y revistas advertían que, de aprobarse un acuerdo migratorio oficial entre Chile y Japón, esta llegaría a ser "una avalancha incontenible por las facilidades que se le habrían dado para atraerla artificialmente y por la abundancia de trabajo bien remunerado [...]. Traer la inmigración amarilla es comprometer por una situación industrial transitoria el porvenir de nuestra raza que merece, sin duda, la atención más preferente" ${ }^{24}$.

21 Allen, Louis. 1987. "Japanese Intelligence Systems," Journal of Contemporary History vol.22, №. 4, p. 548. Cf. lacobelli, 2020. "Reconsiderando la neutralidad chileno-argentina: prensa y diplomacia japonesa durante La Conferencia de Río de Janeiro, 1942."

22 Normano, J. F. 1934. "Japanese Emigration to Brazil," Pacific Affairs 7, no. 1, Vancouer,pp. 42-61,; Beals, Carleton. 1938. "Totalitarian Inroads in Latin America," Foreign Affairs 17, no. 1, ciudad, pp. 78-89; Porter, Catherine.1935. "Japan's 'Penetration' of Latin America," in Far Eastern Survey, vol. 4, №10, New York, pp. 73-78, .Agradecemos a María Catalina Croquevielle por esta información.

${ }^{23}$ Ver por ejemplo, el libro de divulgación Beals, Carleton. 1938. The Coming Struggle for Latin America, Philadelphia, J.B. Lippincott Company.; Everest - Phillips, Max. 2007. "The Pre-War Fear of Japanese Espionage," Journal of Contemporary History 42, no. 2, p. 245.

${ }^{24}$ BN? El Mercurio, Valparaíso, 11 de diciembre de 1912. Citado por Portales, M. 2015. “Cruzando el mar remontando el río: migración e integración japonesa en Chile". Inédito. 
Tanto a principios como a mediados del siglo veinte, los japoneses en Chile eran vistos como inasimilables ${ }^{25}$.

En el contexto de guerra, la información emanada en EE. UU. sobre redes de espionajes del Eje en América del Sur agregó una nueva capa a los resquemores con los que se miraba al japonés. En esta línea, la multiplicidad que parece tener la red de espionaje japonés, en cuanto a quienes participan de la misma y de que formas lo hacen para recopilar la información, llevó a las policías y estados a mantener un estado de alerta constante frente a cualquier actividad que fuera factible de declarar parte de un sistema de espionaje mayor. Estos temores, según Everest-Phillips tienen muchas raíces, manifestándose fuertemente una matriz de prejuicio racial ${ }^{26}$, aspecto de diferenciación que llevará -en el caso chileno- a esgrimir argumentos débilmente sustentados contra Goro Miyazaki.

Este caso no solo refleja los miedos frente a la presencia japonesa en América Latina y Chile, sino también el constante estado de alerta y la reacción frente a situaciones que, desde el punto de vista chileno, eran vistas como fruto de una red de espionaje japonesa. No obstante, la recopilación de fuentes en torno al espionaje es compleja, por la naturaleza misma de la actividad. En este sentido, nuestro trabajo se nutre de fuentes provenientes de archivos aliados y chilenos (la cual se sostenía en información de proveniente de EE. UU.), por lo que nos es difícil llegar a valorar la calidad de espía o agente de Goro Miyazaki; mas no nos impide reconocer que las pruebas en su contra eran superfluas y circunstanciales.

Las fuentes dan cuenta de que la Sociedad Chilena-Oriental, de la cual Miyazaki era fundador y socio mayoritario, fue inspeccionada constantemente, ante la sospecha de que tuviera participación y vínculo en actividades subversivas y de espionaje dentro del territorio nacional ${ }^{27}$. No solo eso, la figura misma de Miyazaki estaba ligada a una serie de reuniones que se realizaban en la legación japonesa, las cuales contaban con la presencia del agregado militar Haruo Tesima y el agregado naval Sadayoshi Nakayama ${ }^{28}$. Para la inteligencia chilena y estadounidense, la vinculación de Miyazaki con el cuerpo diplomático japonés era motivo de sospechas pues se vinculaba a esta en la conformación de redes de espionaje. La legación japonesa fue un centro de inteligencia por cuanto recopilaba información a través de fuentes abiertas tales como periódicos y prensa, al tiempo de vincularse con la clase política local y de esta manera informarse de la situación local como global ${ }^{29}$.

\footnotetext{
25 Estrada, Baldomero. 1997. Presencia Japonesa En La Región de Valparaiso. Un Proceso de Asimilación Étnica y de Desarrollo Agrícola, Valparaíso, Ediciones Universidad de Valparaíso.

${ }^{26}$ Everest - Phillips, Max. 2007. "The Pre-War Fear Of Japanese Espionage", p. 244.

27 Paredes, Mauricio. 2012. Nacionalismo, Seguridad y Presión Internacional: La Relegación de Japoneses En Chile Durante La Segunda Guerra Mundial, p. 451.

28 Ibíd., p. 456.

${ }^{29}$ Allen, L. 1987. “Japanese Intelligence Systems.”, p. 549.
} 
El seguimiento a las acciones de Miyazaki y sus vínculos con el espionaje japonés tuvieron su punto culmine entre julio y agosto de 1942, cuando Goro Miyazaki fue acusado de realizar actividades contrarias a los intereses del país. Si bien esto es mencionado dentro de los argumentos esgrimidos por el Ministerio del Interior frente a la misiva de Miyazaki pidiendo la reconsideración del decreto que lo expulsaba, las razones del Estado chileno se sustentan esencialmente en bases legales, que impedirían de manera irremediable que este ciudadano japonés permaneciera en Chile.

El principal motivo esgrimido por el gobierno de Juan Antonio Ríos para no reconsiderar la expulsión de Miyazaki en agosto de 1942, recae en que el "súbdito" japonés no se habría ajustado a los tiempos correspondientes para solicitar la residencia permanente en Chile, arguyendo que "había sido procedente expulsar del país, sin más trámite, el extranjero mencionado, ya que este permanecía ilegalmente en el territorio de la República y había antecedentes suficientes de que desarrollaba actividades contrarias al interés nacional ${ }^{\prime 30}$. Estos antecedentes refieren a una serie de acciones llevadas a cabo por Miyazaki y de documentos encontrados entre sus pertenencias que lo erigen como un personaje sospechoso. Así, es acusado de tomar fotografías aéreas, durante un viaje a Freirina para establecer lazos comerciales con la Compañía La Cobaltera, las cuales fueron discutidas como posible acto de espionaje pues era plausible de utilizar estas imágenes con fines estratégico-militares por cualquier nación enemiga.

Sumado a lo anterior, durante la primera quincena de mayo de 1942, fue detenido en Arica, acusándole de intentar abandonar Chile y con una serie de documentos en japonés (que por su impenetrabilidad arrojaba sospechas a los funcionarios chilenos de la época). Además, entre sus pertenencias, la policía chilena halló información sobre yacimientos de manganeso y cobalto, considerado de valor estratégico. Los papeles en su lengua materna son objeto de sospecha inmediata, jugando el recelo un rol importante en la inclinación de identificar a un japonés como potencial espía. La investigación policial no continuó por expresa petición de la legación japonesa en Chile. De hecho, en ese momento, según lo destacado por el mismo Miyazaki, el Director General de Investigaciones se comunicó personalmente con él, lamentando las molestias causadas, destacando que las futuras acciones de la Dirección General de Investigaciones se ajustarían a su comportamiento dentro del país ${ }^{31}$.

Semanas antes, otro de los socios de la Sociedad Comercial también era vinculado a lo que el diario El Siglo denominó un "ampliado fascista", llevado a cabo eventualmente entre los días 9 y 16 de abril. El hecho, informado por el diputado y dirigente sindical Salvador Pastene, daba

\footnotetext{
${ }^{30}$ ARNAD, Fondo Ministerio del Interior, Raúl Morales a la Legación del Japón, 8 de septiembre de 1942, v. 10816, Cursivas nuestras.

${ }^{31}$ ARNAD, Fondo Ministerio de Relaciones Exteriores Goro Miyazaki al Ministro del Interior, 28 de agosto de 1942, , v. 4635 ,
} 
cuenta de tres japoneses que se encontraban en la ciudad de Arica al mismo tiempo que llegaban desde Bolivia un grupo de repatriados japoneses, italianos y alemanes. Uno de los ciudadanos japoneses identificado fue Sueyuki Nakatsuka, socio de la Sociedad Comercial Chileno-Oriental, de la cual era representante Miyazaki. Al ser consultado respecto al porqué de su presencia en la mencionada ciudad, su respuesta fue que se encontraba allí con el propósito de tomar contacto con los representantes de su casa comercial en Bolivia ${ }^{32}$. Las acusaciones contra Miyazaki y los vínculos que se establecían con él no acaban allí, en la medida que se le seguían sus pasos por el territorio nacional se le continuó vinculando con actividades subrepticias, que llegaron a trascender en la prensa nacional. Por ejemplo, el semanario Ercilla publicó - sin dar fuentes - sobre la existencia de una organizada quinta columna japonesa, en la cual, junto con miembros de la legación de ese país en Chile, participaba él y tenía como objetivo apropiarse de la producción nacional de salitre ${ }^{33}$.

Nuestra revisión de fuentes permite emplazar la figura de Goro Miyazaki como relevante para el quehacer de la misión japonesa en Chile. Esto debido al fortalecimiento del vínculo comercial entre ambos países y del cual participa activamente Miyazaki a través de la Sociedad Comercial Chilena Oriental y la representación del gigante Mitsui en el país. Empero, tomando en cuenta que Miyazaki fue un prominente ciudadano del Eje en Chile, es comprensible que sus actividades, con el objetivo de aprovechar la riqueza mineral chilena en el fortalecimiento de las relaciones comerciales con el Japón, fuesen vistas como actividades con motivaciones más allá de lo exclusivamente comercial. A esto se suma lo anteriormente planteado, en la medida de que el temor frente a la amenaza japonesa y a un posible ataque a las costas nacionales movilizó al gobierno a tomar acciones concretas, que en algunos casos llevó a tensionar la neutralidad planteada en la Conferencia de Río de Janeiro, en la medida que en reiteradas ocasiones, los técnicos encargados de interceptar comunicaciones radiales, fueron capacitados por personal estadounidense o, explícitamente, personal enviado desde Estados Unidos ${ }^{34}$. Esto implicó que, desde febrero del año 1942, Chile intentara defender y fortificar el litoral contra actividades de sabotaje, contemplando también el bloqueo de señales radiales y el monitoreo de la actividad naval, asunto destacado en numerosos periódicos de la prensa británica ${ }^{35}$,

\footnotetext{
${ }^{32}$ ARNAD, Fondo Ministerio del Interior, informa Providencia $n^{\circ} 147$ de fecha 5 de mayo de 1942, 18 de mayo de 1942 , v. 10788 ,

33 "El Salitre Chileno va al Eje", Ercilla, 12 de agosto de 1942, s/n.

$34 \mathrm{El}$ volumen 4635 del Fondo del Ministerio de Relaciones Exteriores contiene una serie de documentos de solicitudes y comunicaciones entre el Gobierno de Chile y diversos servicios de inteligencia o militares de Estados Unidos, con el propósito de monitorear y detectar redes de espionaje, intervención de cable telegráficos y evitar actividades de sabotaje en las costas chilenas.

35 ARNAD, Fondo Ministerio de Relaciones Exteriores, Departamento diplomático al Ministro de Relaciones Exteriores, "Remite recortes de prensa sobre defensa de la costa chilena", 19 de febrero de 1942, v. 4637.
} 
además de una cooperación constante en términos de seguridad y detección de redes de espionaje.

Considerando lo anterior y el ambiente de alerta frente a cualquier actividad realizada por ciudadanos de los países del Eje, no se comparte en su totalidad la premisa de Mauricio Paredes respecto a que, si "Miyazaki efectivamente fue un espía, su defensa puede haber sido un error que luego pagarían muchos japoneses relegados bajo la acusación de haber sostenido contacto con él" ${ }^{36}$. Si bien la defensa de Miyazaki efectivamente pudo plantear desafíos a la sobrevivencia de la red de espionaje japonesa presente en Chile, las repercusiones para quienes fueron vinculados ya sea con la Sociedad Chilena Oriental o con el mismo Goro Miyazaki fueron, por lo demás, tardías y restringidas tan solo a la relegación. Ocho personas se detectaron hacia 1942 vinculadas con Goro Miyazaki, no obstante, solo fueron relegadas a distintos lugares del país y a poca distancia de Santiago. El único expulsado y acusado derechamente de ejercer actividades de espionaje y contra los intereses del país, con una red de contacto que alcanzaba la legación japonesa y vinculado al rubro comercial, eje de la expansión y consolidación de Japón en Latinoamérica, fue el ciudadano japonés Goro Miyazaki.

Asimismo, si bien la férrea defensa de la Legación Japonesa puede ser vista como poco estratégica, en el sentido de que significaba levantar sospechas sobre otros personajes involucrados con Miyazaki y poner de manifiesto la red de espionaje, se debe reconocer la importancia de Miyazaki en términos comerciales y políticos, por lo que perder a Miyazaki y permitir la acusación de espionaje, implicaba reconocer explícitamente la efectiva existencia de una red dedicada a la recopilación de información estratégica, no solo respecto a la neutralidad chilena, sino también referente a movimientos navales y comerciales vitales para la permanencia de Japón en Latinoamérica. Si bien Paredes arguye que era mejor mantener cierto bajo perfil, según los datos que el mismo autor menciona, en base a los documentos estadounidenses, no obstante, la Legación refiere a que "el señor Miyazaki no es un comerciante cualquiera" ${ }^{37}$. No solo se reconoce la importancia comercial de Miyazaki, sino también es necesario destacar los contactos conocidos a nivel gubernamental ${ }^{38}$, por lo tanto,

\footnotetext{
36 Paredes, Mauricio. 2012. Nacionalismo, Seguridad y Presión Internacional: La Relegación de Japoneses En Chile Durante La Segunda Guerra Mundial, p. 460.

${ }^{37}$ ARNAD, Fondo Ministerio de Relaciones Exteriores, Ernesto Barros al Ministro del Interior, "Transcribe Nota N 18 de la Legación del Japón", 2 de septiembre de 1942, vol. 4635.

38 Paredes, a partir de la revisión de los archivos estadounidenses, puede dar cuenta de esto en su tesis doctoral. Según un reporte confidencial, se pudo dar cuenta de las visitas de Miyazaki al Club de la Unión y el ofrecimiento de negocios y préstamos a distintas personas en cargos de gobierno. Producto de los vínculos con Miyazaki, incluso llegó a ser investigado el expresidente Juan Esteban Montero, por su presunta colaboración para ocultar y reinvertir el dinero de la Sociedad Comercial Chileno Oriental, mediante la compra de propiedades y de una compañía carbonífera en Lota. Paredes, Mauricio. 2012. Nacionalismo, Seguridad y Presión Internacional: La Relegación de Japoneses En Chile Durante La Segunda Guerra Mundial, pp. 459 - 462.
} 
no era algo novedoso la defensa de Miyazaki por parte de la Legación, así como su alto perfil dentro de la comunidad japonesa residente en Chile.

La defensa de la Legación a Miyazaki no es lo único que llama la atención respecto a este caso. La información que en los documentos chilenos se pudo obtener del mencionado ciudadano japonés es proveniente de los Fondos antes citados del Ministerio del Interior y el Ministerio de Relaciones Exteriores. No obstante, al revisar los cuadernos donados al Archivo Nacional por la Policía de Investigaciones (PDI) ${ }^{39}$, pertenecientes al Departamento $50^{40}$, específicamente los cuadernos 15, 16, 19 y 21, que abarcan entre 1941 y 1944, no se encuentra información referente a Goro Miyazaki. Sin embargo, es necesario mencionar un memorándum del 8 de julio de 1942, en el cuaderno 16 del Departamento 50, anterior a la acusación contra Miyazaki, y que vislumbra los primeros vínculos entre las redes de espionaje alemán y la red de espionaje japonés. En este memorándum se devela el vínculo entre "Bach", identificado como Ludwig von Bohlen, con los agregados militares y navales de la Legación de Japón en Chile. Von Bohlen, agregado aéreo de la Embajada de Alemania en Santiago, tenía vínculos con la Compañía de Transportes Marítimos. Los agentes, situados en la mencionada compañía, actuaban como catalizadores de información a través de la estación de radio PYL. No solo eso, además se interceptó un mensaje en donde von Bohlen solicitaba permiso a Alemania para compartir información relativa al movimiento de naves al agregado militar japonés ${ }^{41}$.

La información recopilada a partir de este memorándum permite iluminar los vínculos entre las redes de espionaje de los países del Eje presentes en Chile. Japón, desde la legación, gestionaba la recopilación de información, lo que implicaba hacer cruce de datos con los otros miembros del Eje. Sin embargo, el funcionamiento de espionaje alemán devela además lo similar que resulta con la red de espionaje japonesa, jugando un rol fundamental el posicionamiento de empresas vinculadas al comercio transnacional y las representaciones diplomáticas. Mientras las circunstancias lo permitieron, la neutralidad chilena implicó un espacio de maniobrabilidad para las redes de espionaje. Adicionalmente, el funcionamiento de la red de espionaje alemana, estudiada en extenso por el Departamento 50, permitió

\footnotetext{
39 La Policía de Investigaciones, entre junio y julio del presente año, realizó la transferencia de archivos del Departamento 50 al Archivo Nacional, con el propósito de preservar los archivos originales. Los cuadernos están disponibles para su consulta en línea a través del sitio web del Archivo Nacional.

${ }^{40}$ El Departamento 50, nombre con el que coloquialmente se le conoció, por el anexo telefónico que ocupaba, nace a fines de 1942 como reacción al hallazgo de redes de espionaje alemanas en Chile (PYL y PQZ, estaciones radiales a través de las cuales se transmitía información). La Sección Confidencial Internacional, nombre oficial del Departamento 50, tuvo su origen en una pequeña unidad creada en 1941 encargada de investigar actividades nacionalsocialistas en el sur de Chile. Durante su trabajo, lo hizo en coordinación con el SIS (Special Intelligence Service), unidad creada por el FBI para investigar el nazismo en América Latina.

${ }^{41}$ Memorándum para el Embajador. Referente: Agentes del espionaje alemán en Chile. Cuaderno 16, Dirección General de Investigaciones e Identificación, Departamento 50, Archivo Nacional de Chile, 30 de junio de 1942, foja 237. Disponible en: http://www.archivonacional.cl/sitio/Contenido/Institucional/82968:Cuadernos-del-11-al-23.
} 
comprender y extrapolar su funcionamiento a otras redes de espionaje, en este caso, la japonesa. Esto habría llevado a poner el foco en las actividades de Goro Miyazaki.

Sin embargo, esto no implica que la tarea de poner de relieve redes de espionaje haya sido una labor eminentemente chilena. Tal y como lo destacan las fuentes utilizadas por Mauricio Paredes y asimismo lo reconocido en múltiples ocasiones en el mismo memorándum por la inteligencia chilena, gran parte de los primeros datos respecto a la red de espionaje alemán, sus vínculos con el espionaje japonés y, fundamentalmente, los vínculos de Miyazaki en Chile, provienen desde los aparatos de inteligencia estadounidense: la utilización de empresas como pantalla para desarrollar actividades de espionaje era algo que Estados Unidos ya venía investigando. Lo anterior da cuenta de lo dependiente que resultan los servicios de inteligencia chilenos al momento de enfrentarse a las redes de espionaje. Tanto en los cuadernos 15 y 16 del Departamento 50 en reiteradas ocasiones hacen referencias a que las informaciones provienen desde el Departamento de Estado de los Estados Unidos, en base a lo cual se orientaban las directrices de investigación en Chile. Esto se inserta dentro del posicionamiento que Estados Unidos adoptó respecto a América Latina luego del ataque japonés, especialmente ampliando su presencia a través de bases militares en el frente occidental y Latinoamérica, con la intención de frenar la intromisión de agentes del $\mathrm{Eje}^{42}$. Cabe mencionar, que dada la revisión de los cuadernos del Departamento 50 y de los Fondos del Ministerio del Interior y de Relaciones Exteriores, que para Estados Unidos no solo era relevante el posicionamiento en términos militares, sino también la circulación de información y de personas, puntos hacia donde se enfocó la tarea en inteligencia, sobre todo en el territorio nacional.

Respecto a la situación de Miyazaki, el gobierno chileno desestimó finalmente los argumentos entregados, tanto por Miyazaki, así como por la Legación Japonesa en Chile. Se decantó por la opción de considerarlo un agente peligroso para la seguridad nacional e involucrado en actividades de espionaje, manteniendo la resolución de expulsión manifestada en el Decreto $\mathrm{N}^{\circ} 4097$, publicado en el Diario Oficial ${ }^{43}$.

Al momento de la expulsión de Goro Miyazaki, las relaciones chileno-japonesas enfrentaban lo que César Ross ha denominado la fase de caída y declive de los vínculos entre ambas naciones, especialmente entre 1941 y 1943, momento de la ruptura definitiva de las relaciones con Japón ${ }^{44}$. Consideradas estas circunstancias y lo planteado con anterioridad a lo largo del texto, el caso de este ciudadano japonés en particular resulta interesante de analizar, en la medida que entrega aproximaciones respecto al estado de las relaciones diplomáticas de Chile

\footnotetext{
42 Pettyjohn, Stacie. 2012. "Hemispheric Defense, 1938 - 1941," en U.S. Defense Posture, 1783 - 2011 (RAND Corporation, pp. 39-48.

43 ANH/BN? Ministerio del Interior, Decreto N4097, "Deniega lugar a la solicitud presentada por el ciudadano japonés Goro Miyazaki Miyazaki, en que pide permiso para permanecer indefinidamente en el país", Diario Oficial de la República de Chile, ejemplar N 19.332, 12 de agosto de 1942, p. 11.

${ }^{44}$ Ross, César. 2013. "Auge y Caída de Japón En Chile, 1897 - 1943", p. 170.
} 
con Japón. Al mismo tiempo, permite dar cuenta de que manera el Estado de Chile y el sistema latinoamericano reaccionó frente a las prácticas de espionaje, en pleno desarrollo de la Segunda Guerra Mundial, situación vinculada estrechamente con los servicios de inteligencia estadounidenses. Por último, implica considerar la neutralidad chilena aplicable plenamente en un sentido estrictamente diplomático y bélico, pero en relación con la circulación de información y personas, Chile tomó una posición clara, precisa y comprensible dentro del contexto global.

\section{Conclusión}

El año 1942 fue convulsionado a nivel nacional y latinoamericano. La inseguridad frente a la presencia japonesa -y en general frente las comunidades de ciudadanos de los países del Ejemarcó el transcurso del año en lo político, social y defensa, al mismo tiempo que determinó el trato hacia estas comunidades e individuos en general. El caso de Goro Miyazaki se inserta dentro de este contexto, de un ambiente tensionado por la sensación de amenaza y de que había individuos al interior del territorio chileno proclives a atentar contra la seguridad nacional. El ataque de Japón a Pearl Harbor a fines de 1941 despertó un profundo espíritu de solidaridad entre los países latinoamericanos, coronado en el caso chileno, con el fin de su neutralidad y rompiendo relaciones con Japón a inicios de 1943.

La ruptura de relaciones fue un proceso no exento de discusiones y divergencias a nivel nacional. El estado del espíritu nacional respecto a la posición de Chile en la guerra puede seguirse durante fines de 1941 y todo el año 1942 a través de las sesiones de las cámaras de Diputados y Senadores. La neutralidad y la investigación de actividades nacistas (extendidas a toda actividad sospechosa realizada por los ciudadanos de los países del Eje) marcan la pauta en diversas sesiones, tanto ordinarias como extraordinarias ${ }^{45}$. La posición del gobierno, encabezado por el presidente Juan Antonio Ríos, de mantener la neutralidad, despertó rivalidades y discusiones en el Congreso $\mathrm{Nacional}^{46}$, dando cuenta de la tensión existente a nivel político.

\footnotetext{
45 En la sesión 2. a de la cámara de Diputados se discutió la creación de una Comisión Investigadora de actividades nacistas. La discusión en el momento transcurrió entre si plantearlo como "nacista" o cambiar la nominación por "elementos extranjeros" (postura del diputado conservador Marín Balmaceda y apoyado por el comité liberal). Sesión Ordinaria de la Cámara de Diputados de Chile, 26 de mayo de 1942, Biblioteca Nacional de Chile.

${ }^{46}$ En la sesión 4.a de la cámara de Diputados, el diputado Zepeda (Partido Liberal), responde a una alusión hacia su persona en el diario El Siglo, criticando su nominación como miembro de la Comisión Investigadora. Acusa que el diario hace uso de la injuria y la calumnia, debido a que se le ha catalogado como espía nazi, cuya designación no hará más que entorpecer la labor de la comisión. El diputado acusa a los comunistas de no ser chilenos, sino "internacionales o, mejor dicho, rusos". La sesión termina con insultos varios entre los diputados, tratándose de "enfermos mentales". Sesión Ordinaria de la Cámara de Diputados de Chile, 1 de junio de 1942, Biblioteca Nacional de Chile.
} 
A pesar de esa neutralidad declarada, los documentos cotejados dan cuenta de que aquella neutralidad fue transgredida en la medida que gran parte de la información que poseían los servicios de inteligencia chilenos al momento de perseguir y emitir juicios sobre la calidad de espía o no de un individuo, provenía de los servicios de inteligencia estadounidenses. Así, la expulsión del ciudadano japonés Goro Miyazaki responde, en gran medida, a la influencia de la información emitida desde Estados Unidos sobre los servicios de inteligencia chilenos, todo esto parte de una red coordinada para detectar las redes de espionaje de los países del Eje en Latinoamérica.

En general, a principios de la década de 1940, existe un contexto global que permea la sociedad chilena y que se vio reflejada en las relaciones sociales, políticas y diplomáticas que se establecieron con los países del Eje Roma - Berlín - Tokio. Los florecientes vínculos comerciales, que alcanzaron su máximo esplendor hacia fines de la década de 1930, se vieron seriamente perjudicados por los avatares de la guerra. Demostración de esto es la actitud que el gobierno de Chile tomó contra las acciones de Miyazaki, a pesar de los infructuosos intentos por presentar su rol como representante comercial en defensa del ciudadano japonés.

Por otra parte, es importante relevar lo central que era Goro Miyazaki, al menos en términos comerciales, para la comunidad japonesa residente en Chile para la época. Si bien otros miembros fueron más destacados y reconocidos dentro de la sociedad santiaguina, Miyazaki era representante de una de las firmas más grandes de Japón y a través de la cual se consolidaban los nexos con América Latina. Sumado a lo anterior, Miyazaki construyó sólidos vínculos con la legación japonesa en Chile, encabezada hacia 1942 por el ministro Yamagata. No obstante, independiente de sí Miyazaki fue o no espía y considerando la defensa esgrimida por la legación, el caso de Goro Miyazaki refleja que la hostilidad hacia los japoneses en general fue superficial, circunscribiendo las actividades de espionaje y las sospechas relacionadas con esta, al rol comercial que cumplía este ciudadano japonés.

Así, el caso Miyazaki, como lo hemos denominado, da cuenta del espíritu de la época y las tensiones que, a través de la experiencia de un sujeto en particular, nos permite visualizar las dinámicas y aspectos claves para comprender el impacto de lo global en lo local y los vínculos que se establecen entre los distintos espacios y personas. De esta forma, la guerra ya no solo puede ser concebida como exclusivamente europea, sino que en Goro Miyazaki encontramos ese cruce entre la experiencia japonesa en Chile y Latinoamérica, la coyuntura crítica de la Segunda Guerra Mundial y las tensiones evidentes de un Chile que debía ser consecuente y mantener a raya las actividades de la "Quinta Columna", cualesquiera fueren sus manifestaciones. 


\section{Fuentes y Bibliografía}

\section{Fuentes}

Archivo Nacional de Chile (s.f.). Dirección General de Investigaciones e Identificación. Departamento 50, Cuaderno 16. Disponible en:

http://www.archivonacional.cl/sitio/Contenido/Institucional/82968:Cuadernos-del-11-al-23.

Archivo Nacional de la Administración (ARNAD). Fondos del Ministerio del Interior y Relaciones Exteriores, 1942.

Biblioteca Nacional de Chile. s.f. Sesiones Ordinarias de la Cámara de Diputados, 1942.

Diario El Siglo, 1942.

Revista Ercilla, 1942.

Estatutos Del Instituto de Cultura Chileno Japonés. 1940. Santiago, Imprenta y Litio

Instituto Nacional De Estadísticas. Anuario Estadístico del Comercio de Chile. Años 1930-1941.

\section{Bibliografía}

Abel, Jessamyn Reich. 2013. "Cultural Internationalism and Japan's Wartime Empire: The Turns of the Kokusai Bunka Shinkōkai.", en Tumultus Decade: Empire, Society, and Diplomacy in 1930's Japan, Toronto, University of Toronto Press, pp. 17-43.

Allen, Louis. 1987. “Japanese Intelligence Systems", en Journal of Contemporary History 22, no. 4, Londres, pp. 547-62.

Barros V, Mario. 1998. La Diplomacia Chilena En La Segunda Guerra Mundial, Santiago, Empresa Editora Arquen.

Beals, Carleton. 1938. The Coming Struggle for Latin America, Philadelphia, J.B. Lippincott Company.

Beals, Carleton. 1938. "Totalitarian Inroads in Latin America", en Foreign Affairs 17, no. 1, Nueva York, pp. 78-89. doi:10.2307/20028904.

Braun-Llona, Juan, Braun-Llona, Matías, Briones, Ignacio, Diaz, José, Lüders, Rolf, and Gest Wagner. 1998. Economía Chilena 1810-1995. Estadísticas Históricas, (Serie: Documentos de Trabajo No. 187), Santiago, Facultad de Economía Pontificia Universidad Católica de Chile

Castellón, Alvaro, y Pozo, José Manuel. 1997. “Historia de Las Relaciones Bilaterales Chile y Japón”, en Pinochet de la Barra, Oscar (ed.), Chile y Japón Un Siglo de Amistad, Santiago, Comisión Chilena de Celebración del Centenario de las Relaciones Chile-Japón, pp. 129 - 160.

Estrada, B. 1997. Presencia Japonesa En La Región de Valparaíso. Un Proceso de Asimilación Étnica y de Desarrollo Agrícola, Valparaíso, Ediciones Universidad de Valparaíso.

Everest Phillips, Max. 2007. "The Pre-War Fear of Japanese Espionage", en Journal of Contemporary History 42, no. 2, Londres, pp. 243-65.

Farías, Víctor. 2000. Los Nazis En Chile, Hong Kong, Editorial Wide Chance.

Fermandois, Joaquín. 1988. "Guerra y Hegemonía 1939-1943. Un Aspecto de Las Relaciones ChilenoNorteamericanas", en Historia 23, no. 1, Santiago, pp. 5-51.

Ferrando, M. Teresa. 2004. Al Otro Lado Del Pacífico: Japoneses En Chile, 1900 - 1960, Santiago, Ograma. Gaimushō Ryōji lijūbu. 1971. Waga kokumin no kaigai hatten: ijū hyakunen no ayumi (honhen)", Tokio, Ministry of Foreign Affairs.

Hachette, Dominique. 1997. "Relaciones Económicas Entre Chile y Japón." en Pinochet de la Barra, Oscar (ed.), Chile y Japón Un Siglo de Amistad, Santiago, Comisión Chilena de Celebración del Centenario de las Relaciones Chile-Japón, pp. 163-199. 
lacobelli, Pedro. 2016. “La 'Neutralidad’ Chilena En La Segunda Guerra Mundial (1939-1943): Un Análisis

Historiográfico Con Énfasis En La Literatura Sobre Las Relaciones Chile-Japón.", en Revista de Historia y Geografía 34, Santiago, pp. 95-108.

lacobelli, Pedro. 2017. Postwar Emigration to South America from Japan and the Ryukyu Islands, London,

Bloomsbury Academic.

lacobelli, Pedro. 2020. "Reconsiderando La Neutralidad Chileno-Argentina: Prensa y Diplomacia Japonesa

Durante La Conferencia de Río de Janeiro, 1942", en Ayer, vol. 119, no. 3, Madrid, pp. 137-163.

lacobelli, Pedro. 2020. "Conservadores, Radicales y el "interés nacional": las redes de apoyo al Imperio de Japón en Chile durante la Segunda Guerra Mundial”, en Izquierdas, No. 49 (Mayo), Santiago, pp. 3242-3260.

lacobelli, Pedro, y Camino, Nicolás. 2018. “Diplomacia Pública Japonesa en La Prensa Chilena Durante La

Segunda Guerra Mundial", en Cuadernos de Historia. 42, no. 2, Santiago, pp. 73-97.

Kotani, Ken. 2009. Japanese Intelligence in World War II, Oxford, Osprey Publishing.

Matthews, T. n.d. Spies for Nippon: Japanese Espionage Against the West, 1939-1945, Londres, Robert

Hale \& Company.

Nocera, Raffaele. 2006. Chile y La Guerra 1933-1943, Santiago, LOM editores.

Normano, João Frederico. 1934. "Japanese Emigration to Brazil”, en Pacific Affairs 7, no. 1, Vancouver, pp. 42-61. doi:10.2307/2750689.

Paredes V, Mauricio. 2012. Nacionalismo, Seguridad y Presión Internacional: La Relegación de Japoneses En Chile Durante La Segunda Guerra Mundial, Santiago, Universidad de Chile.

Pettyjohn, Stacie. 2012. "Hemispheric Defense, 1938 - 1941" In U.S. Defense Posture, 1783 - 2011, Santa Mónica, CA, RAND Corporation. pp. 39-48.

Porter, Catherine. 1935. “Japan's 'Penetration' of Latin America”, en Far Eastern Survey 4, no. 10, Berkeley CA, pp. 73-78. doi:10.2307/3021468.

Rodao G, F. 2012. Franco y El Imperio Japonés, Barcelona, Plaza y Janes.

Ross, César. 2013. "Auge y Caída de Japón En Chile, 1897-1943”, en Estudios Políticos 43, Santiago, pp. 156-179.

Ross, César. 1999. “Chile y Japón: Balance de Un Siglo de Relaciones Económicas, 1897-1997”, en Revista Diplomacia 78, Santiago.

Takeda M, Ariel. 2002. "Japanese Immigrants and Nikkei Chileans", en Akemi Kikumura-Yano (ed), Encyclopedia of Japanese Descendants in the Americas, Walnut Creek, CA, AltaMira Press. 